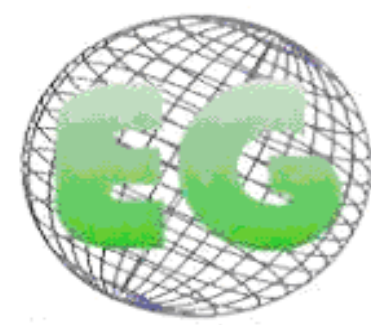

ISSN 1695-6141 N'25

\title{
PINCHAR... ¡HACE DAÑO! Representaciones del dolor en el niño, en edad escolar,sometido a punción venosa
}

PICAR... FAZ DOER! Representações de dor na criança, em idade escolar, submetida a punção venosa

\section{${ }^{*}$ Guerreiro, MR., ${ }^{* *}$ Curado, MA}

\author{
*Enfermeira, Hospital de Faro, EPE. ** Professora Coordenadora, Escola Superior de Enfermagem de \\ Lisboa. Portugal.
}

Palavras chave: criança; idade escolar; dor; punção-venosa; representações-sociais

Palabras clave: niño; edad escola; dolor; punción venosa; representaciones sociales.

Keywords: child; school-age; pain; venous-puncture; social-representations

\section{RESUMEN}

Introducción: El dolor forma parte de la condición humana y es inalienable de su existencia. Es entendido como una experiencia universal con inicio precoz en cada individuo y gana heterogeneidad de configuraciones sociales y variabilidad de grados de intensidad. Como experiencia intransmisible, marcará, de diversas formas, la construcción psicológica y social de la persona. En el niño es una experiencia común y perturbadora, muchas veces subestimada y subintervencionada en contextos de salud.

Metodología: Con este estudio exploratorio de naturaleza mixta, basado en la teoría de las representaciones sociales, pretendemos dar voz a los niños sometidos a punción venosa.

Los objetivos de este trabajo son identificar las representaciones asociadas a la experiencia de punción venosa y evaluar el grado de dolor asociado a esta experiencia, en niños en edad escolar. Se aplicó la asociación libre de palabras con dos cuestiones estímulo: «El dolor me hece pensar en...», "El pinchazo de la aguja me hace sentir...» y la escala numérica de evaluación del dolor, a 43 niños con edades comprendidas entre 6 y 12 años ingresados en un hospital de la Sub-región de Salud de Lisboa. Los datos se analizaron con recurso del software SPAD-T (análisis factorial de correspondencias simples) y SPSS (estadística descrpitiva).

Resultados: Los datos obtenidos permiten decir que los niños que participaron en este estudio consideraron que el dolor funciona como factor desencadenante de sufrimiento y está asociado a manifestaciones físicas como el llanto y a sentimentos expresados ante el dolor, como el miedo y la ansiedad. El grado de dolor está asociado a la punción venosa y presenta variabilidad con factores como la edad y las experiencias anteriores.

Conclusión: La punción venosa es un procedimiento doloroso y, según los niños que participaron en el estudio, provoca miedo y ansiedad. Sin embargo, muchos evitaron tener comportamientos sugestivos de dolor por vergüenza de ser considerados más flojos o por entender que es un dolor necesario. De ahí el hecho de que algunos niños, asociaran al dolor la valentía que se necesita tener para soportarla. 


\section{RESUMO}

Introdução: A dor faz parte da condição humana e é inalienável da sua existência. É entendida como uma experiencia universal com início precoce, em cada indivíduo e ganha heterogeneidade de configurações sociais e variabilidade de graus de intensidade. Como experiência intransmissível, irá marcar, de diversas formas, a construção psicológica e social da pessoa. Na criança é uma experiencia comum e perturbadora, muitas vezes subestimada e subintervencionada em contextos de saúde.

Metodologia: Com este estudo exploratório de natureza mista, baseado na teoria das representações sociais pretendemos dar voz às crianças submetidas a punção venosa. Os objectivos deste trabalho são identificar as representações associadas à experiencia de punção venosa e avaliar o grau de dor associado a esta experiencia, em crianças em idade escolar. Foi aplicada a associação livre de palavras com duas questões estímulo: «Dor faz-me pensar em....», «A picada da agulha faz-me sentir...» e a escala numérica de avaliação da dor, a 43 crianças com idades compreendidas entre 6 e 12 anos internadas num hospital da Sub-região de Saúde de Lisboa. Os dados foram analisados com recurso ao software SPAD-T (análise factorial de correspondências simples) e SPSS (estatística descritiva).

Resultados: Os dados obtidos permitem dizer que as crianças que participaram neste estudo consideraram que a dor funciona como factor desencadeante de sofrimento e está associado a manifestações físicas como o choro e a sentimentos expressos perante a dor, como o medo e ansiedade. $\mathrm{O}$ grau de dor associado à punção venosa e apresenta variabilidade com factores como a idade e as experiencias anteriores.

Conclusão: A punção venosa é um procedimento doloroso e, segundo as crianças que participaram no estudo, provocam medo e ansiedade. No entanto, muitas evitarão ter comportamentos sugestivos de dor por vergonha de serem consideradas mais fracas ou por entenderem que é uma dor necessária. Daí o facto de, algumas crianças, associarem a dor à coragem que é necessário ter para suportá-la.

\section{ABSTRACT}

Introduction: Pain is a part ofhuman nature and is an unalienable part of our existence. It is understood as a universal experience, with an early beginning, in each person, and it gans heterogeneity from social configurations and varying intensity. As an untransferrableexperience, it marks the construction of the psychological and social person in various ways. For children, pain is a common and disturbing experience that is sometimes underestimated in health contexts.

Methodology: This exploratory study is of a mixed nature, which was based on the theory of social representations, aims to dentify the representation associated to pain, in school age children submitted to venous puncture and to the degree ofpain associated to venous puncture. Therefore, the free association of words technique was applied using two stimulus questions: «Pain makes me think about...», the needle prick makes me feel...», and the numerical scale of pain evaluation, to forty three children with ages between six and twelve years old, who were hospitalized in Lisbon's Health Sub-region. The data have been analyzed using SPAD-T (factorial analysis of simple correspondence) and SPSS (descriptive statistics) software.

The Results show that for those children pain is a factor which arouses suffering and is associated, by children, to physical manifestations, such as crying and to feelings expressed in the presence of pain, like fear. This procedure provokes fear and anxiety in hospitalized children. The degree of pain associated to venous puncture changes with factors like age and mainly with previous experience.

In Conclusion, venipuncture is a painful procedure and according to the children who participated in the study, it causes fear and anxiety. However, many avoided suggestive feelings of pain, because they were ashamed to be considered weaker or deem it a necessary pain; hence the fact that some children associate pain and the need to have the necessary courage to support it.

\section{INTRODUCCIÓN}

El niño, en cuanto representante primordial de la persona humana, constituye cada vez más la "brújula social" por la que se orientan sociólogos, antropólogos, psicólogos, biólogos y, en una perspectiva de salud, enfermeros, médicos y otros profesionales. De este modo, con la intención de acompañar más de cerca su evolución en el mundo moderno, urge observar 
con atención sus necesidades, ya que es en la infancia cuando todo el ciclo vital comienza a delinearse.

La investigación en el área del dolor se toma al considerar que este puede tener efectos extremadamente nefastos en el ser humano, principalmente en el niño, y que esta dimensión es, muchas veces, relegada a un segundo plano, dado su carácter subjetivo y de difícil evaluación. La pertinencia de abordar este tema por la Enfermería actual se une al hecho de ser competencia del enfermero generalista atender los aspectos relacionados con el dolor. Por otro lado, al hacerse visible a través de su evaluación, se consigue realzar la importancia de establecer estrategias terapéuticas adecuadas a su control, lo que contribuirá, decisivamente, a mejorar la calidad de vida de los enfermos y reducir la morbilidad, sobre todo, en lo que se refiere al dolor agudo, cuyo control es un deber ético de los profesionales de salud, un derecho de los enfermos y una condición indispensable y para la necesaria humanización de los cuidados.

El dolor es una experiencia común y perturbadora en el niño, que, a veces, es subestimada y subintervencionada en los contextos de salud ${ }^{1}$. La falta de comprensión de los profesionales de salud en lo que respecta al dolor en el niño hace que este prefiera sufrir en silencio, generando sentimientos de ansiedad y miedo desproporcionados a la situación, lo que podrá conducir a estados más graves de ansiedad y terror. Para que se comprenda bien el proceso de dolor en el niño y para que se proceda a una correcta evaluación, es fundamental tener en cuenta su edad, toda vez que las manifestaciones cara a la experiencia dolorosa serán siempre diferentes, de acuerdo con el estadio de desarrollo del niño ${ }^{2}$. De este modo, sabemos que un lactante reaccionará breve pero intensamente al estímulo doloroso a través de un llanto fuerte, movimientos corporales y expresiones faciales características. El toddler manifestará su miedo de forma más o menos expansiva realizando movimientos corporales más específicamente localizados en la región dolorosa, acompañados de verbalizaciones de desconsuelo. El niño puede también mostrarse irascible, hiperactivo e inquieto o, por el contrario, reducir su actividad espontánea, evitando saltos y alterando sus patrones de sueño y alimentación. En esta fase las experiencias dolorosas son altamente ansiogénicas.

A partir de los cuatro, cinco años, el niño comienza a ser capaz de discriminar las diferentes sensaciones, por lo que más fácilmente se conseguirá percibir las reacciones inherentes al miedo y al dolor. Estas sufrirán modificaciones durante este período, traduciendo comportamientos complejos y polifacéticos que dependerán del concepto de dolor del niño, del significado atribuido a la experiencia dolorosa y de sus estrategias de coping. Las expectativas culturales comienzan también a evidenciarse. Por ejemplo, el papel estereotipado de que los "hombres no lloran" es común en pequeños en este grupo de edad, que intentaron ser fuertes y animosos. Las reacciones al miedo, stress y dolor en esta edad se traducen en la agresión dirigida al "responsable del dolor", expresiones verbales de desagrado o intento de disuasión y de dependencia, representando la regresión a modos de comportamiento más reconfortantes. Con todo, el autocontrol en relación a las situaciones dolorosas es creciente. Las quejas psicosomáticas comienzan a manifestarse durante este período, principalmente cuando el niño percibe que podrá recibir más atención o mimos en el papel de enfermo ${ }^{2,3,4}$.

El niño en edad escolar demostrará, ciertamente, menos preocupación por el dolor en sí, que por la incapacidad que pueda derivarse de él, o del propio procedimiento que se va a realizar, dado que su desarrollo cognitivo ya le permite tener noción de la importancia de ser y estar sano, de las consecuencias de enfermar y del significado de la muerte. La técnica más frecuentemente utilizada como ansiolítica por los niños en este estadio de desarrollo es la busca de información acerca de lo que irá a pasar, lo que les da sensación de control. 
Generalmente, el niño ya adquirió métodos pasivos que le permiten enfrentarse al malestar, como es el caso de la distracción del foco de dolor, por lo que es particularmente importante estar atento a los indicios no verbales ${ }^{5}$.

La enfermedad de un niño, principalmente la que requiere hospitalización, representa para la familia un momento de crisis. Es común que desaparezca el papel protector de los padres, a sus ojos y a los del niño, ante la necesidad de aceptar las decisiones de los profesionales, detentores del saber, por lo que es indispensable la disponibilidad de los enfermeros para escucharles, aclarar dudas y ayudar en la reorganización de la familia dentro de la nueva situación y contexto. En la situación de enfermedad, y principalmente de sufrimiento, provocado por el dolor, la familia se siente, generalmente, impotente. El choque inicial alterna con períodos de culpabilización, pánico y agitación, por lo que si no recibieran el soporte adecuado pueden caer en una situación de desorganización y depresión ${ }^{4}$.

Teniendo conocimiento de estos hechos, es responsabilidad del enfermero, en cuanto prestador de cuidados, intervenir a todos los niveles de forma a minimizar el sufrimiento del niño y de su familia, privilegiando los cuidados en asociación con esta, con el objetivo de alcanzar el máximo bienestar del niño y optimizar la capacidad de la familia de interactuar e intervenir con el niño, durante el tiempo en que esta está sometida a niveles elevados de stress, como sucede cuando siente dolor. Case ${ }^{6}$ refiere incluso que "los cuidados centrados en la familia, prestados en asociación con esta, son la filosofía de la enfermería pediátrica de la década de los noventa. Las creencias y valores que sustenta esta filosofía incluye el reconocimiento de que los padres son los mejores prestadores de cuidados para el niño" $(\text { p.193) })^{6}$ De acuerdo con este modelo, el enfermero prestaría solo cuidados técnicos especializados, incentivando a los padres u otros familiares significativos a prestar los cuidados de soporte de las necesidades básicas del niño, interviniendo solo cuando esté comprobado que la familia no tiene las capacidades o conocimientos necesarios para garantizar la eficacia de estos mismos cuidados. La autora concluye refiriendo que "(...) para preservar el crecimiento y el desarrollo del niño, los cuidados a este deben desarrollarse sobre la forma de protección, estímulo y amor. De esta manera, nadie mejor que los padres para garantizarlo" (p.185) ${ }^{6}$.

De esta forma, la intervención inicial de enfermería, junto al niño con dolor, es la evaluación del nivel de ese dolor. Una vez identificado, deberá implementar estrategias para tratarlo. Estas intervenciones pasan por enseñar al niño y a la familia técnicas de alivio del dolor, utilizando, en conjunto, si fuese necesario, terapéutica farmacológica. La implicación de los padres es central en el control del dolor pediátrico. Cuando estos son informados y saben lo que hacer, además de sentirse mucho menos impotentes, transmiten sensaciones de seguridad y protección al niño. Las sugerencias de los padres sobre la forma de aliviar el dolor a su hijo son preciosas y nadie mejor que ellos para llevarlas a la práctica, por lo que los profesionales de salud deben reforzar ese papel y dar siempre la oportunidad de que estas estén presentes en la realización de técnicas que puedan provocar dolor o malestar ${ }^{7,8}$

\section{La representación del dolor en el niño como objeto de estudio}

El campo de estudios de las representaciones sociales es, actualmente, un campo inmensamente fecundo. En este sentido, muchos son los autores que procuran aclarar y establecer los contenidos simbólicos de múltiples objetos de estudio. Entre los estudios más conocidos se encuentran las representaciones sociales de la enfermedad mental, de la violencia, de la salud y de la enfermedad o de la pobreza en medio urbano ${ }^{9,10}$. 
Pero para que no se caiga en la exageración de establecer todo y cualquier fenómeno como objeto de estudio de representaciones sociales es necesario obtener respuesta a la siguiente pregunta:

¿Existen representaciones sociales de todo? Parte de la respuesta a esta pregunta es dada por Sá ${ }^{11}$ al referir que "(...) el hecho de que las representaciones sociales estén estructuradas indica (...) que no hay motivo para que exista una representación social para cada objeto que se pueda pensar. Determinados objetos darán lugar solo a una serie de opiniones y de imágenes relativamente desconexas. Esto también indica que no todos los grupos o categorías sociales tengan, necesariamente, que participar en una determinada representación (...). Es posible, por ejemplo, que un grupo tenga una representación social de cierto objeto y que otro grupo tenga solamente un conjunto de opiniones e informaciones o de imágenes acerca de ese mismo objeto, sin que eso suponga la existencia de una representación social" (p.46) ${ }^{11}$.

Esta noción fue inicialmente propuesta por Moscovici ${ }^{12}$ al determinar las condiciones que afectan a la aparición de una representación social en un determinado grupo. Estas serían la dispersión de la información, la focalización y la presión a la inferencia. De este modo, se comprende que la forma y la intensidad de tales condiciones pueden variar ampliamente de un objeto a otro, dentro de un grupo, así como de un grupo a otro, en relación al mismo objeto $^{12}$. En otro sentido, las representaciones, en cuanto modalidades de pensamiento, son "(...) alguna cosa que emerge de las prácticas en vigor en la sociedad y en la cultura que las alimenta, perpetuándolas o contribuyendo a su propia transformación como tal. Para que un objeto genere una representación social, este debería tener «relevancia cultural» y «espesor social», siendo que estas características pueden ser traducidas si el objeto en cuestión se encuentra implicado, de forma consistente en una práctica de grupo incluyendo la conversación y la exposición a los medios de comunicación socio" (p.50) ${ }^{11}$. De esta forma, para definir un objeto de estudio debe tenerse presente que la representación será un saber efectivamente practicado, no solo una suposición. Deberá ser un fenómeno detectado en comportamientos y comunicaciones que sucedan de hecho. Caso contrario, cabe la posibilidad de tener como resultado una pseudo-representación, emergente de la investigación y no de la práctica del grupo investigado ${ }^{11}$.

Una vez respondida la pregunta inicial importa legitimar el dolor en el niño como objeto de estudio en representaciones sociales. El dolor es una condición indisociable de la popia existencia del Hombre y, aunque sea singular para quien la siente, como cualquier experiencia humana, su significado puede ser compartido, volviendo a una realidad colectiva, pese a que jamás se pueda garantizar que el significado que un individuo le atribuyó corresponda, exactamente, al que otro le da. De esta forma, puede afirmarse que el dolor no prescinde de su dimensión social, por lo que su singularidad como experiencia subjetiva le convierte en un campo privilegiado para estudiar la relación entre el individuo y la sociedad, dado que la experiencia individual se inscribe en un campo de significaciones colectivamente elaboradas, o sea, los individuos solo construyen el significado de sus experiencias, incluyendo la del dolor, mediante referencias colectivas.

Las experiencias vividas por los individuos, su modo de ser, de sentir o de actuar están íntimamente ligados a la sociedad a la que pertenecen, ya que traducido y aprendido subjetivamente, el significado de toda experiencia humana es siempre elaborado histórica y culturalmente, transmitiéndose por la socialización, iniciada al nacer y renovada a lo largo de la vida ${ }^{13,14}$. Así, puede afirmarse que a nivel de sentido común, en todas las culturas y grupos sociales existen conceptos, símbolos o imágenes asociados al dolor. Los individuos buscan en su memoria estos conceptos para combinarlos y reproducirlos en el mundo exterior, a través de diferentes prácticas e imágenes concretas. De esta forma, se constituye 
el proceso de objetivación, que está en la génesis de la representación social del dolor. El anclaje es otra de las operaciones que se encuentra en el origen de las representaciones sociales y que corresponde al modo como los individuos almacenan conceptos nuevos y los relacionan con objetos y eventos ya conocidos. Es a través de estos dos procesos como se forman y transforman las representaciones sociales del dolor. Una vez formadas, estas constituirán una forma de conocimiento práctico, socialmente elaborado y compartido, basándose en la cual los grupos sociales construyen o adaptan a su propia realidad ${ }^{9,15}$.

En este sentido, el objetivo general definido para este trabajo es: conocer las representaciones del dolor en los niños, en edad escolar, que hayan sido sometidas a punción venosa y, como objetivos específicos: 1) identificar las representaciones asociadas a la palabra dolor, en niños en edad escolar, sometidas a punción venosa; 2) identificar las representaciones asociadas a experiencia de punción venosa, en niños de edad escolar; 3) evaluar el grado de dolor asociado a punción venosa, en niños en edad escolar.

\section{METODOLOGÍA}

Los métodos de investigación se basan en la determinación de procedimientos sistematizados para la definición y explicitación de fenómenos, consistiendo en la delimitación de un problema, en la observación e interpretación de las relaciones encontradas, por lo que se verifica una proximidad al proceso utilizado en el método científico. Los autores que realizaron estudios en el campo de las representaciones sociales refieren que una de las cuestiones levantadas en la discusión metodológica en la investigación en representaciones sociales es la dicotomía entre los abordajes cuantitativos y cualitativos, siendo "(...) esta distinción improductiva cuando impone una elección entre dos posiciones radicales y "monoteístas" (p.64) ${ }^{16}$. Así, optamos por un estudio mixto, pues consideramos que una estrategia multimétodo permitirá analizar, más globalmente, las dimensiones de una determinada representación, garantizando una interpretación integrada de los resultados y de las técnicas. El objetivo es que esta propuesta no sea considerada como una simple suma de métodos, sino que posibilite la selección de los mismos en función de los fenómenos que se pretenden estudiar ${ }^{16}$, toda vez que las variables en estudio son cualitativas (el estímulo que dará origen a la asociación libre de palabras), sin embargo, el tratamiento de los datos será realizado a través de un software de análisis factorial y la técnica auxiliar utilizada será una escala de evaluación del dolor, lo que remite a un abordaje más cuantitativo.

\section{Instrumento de Recogida de Datos}

Las técnicas de investigación se destinan a la recogida de datos, siguiendo determinados criterios y contingencias de modo que los elementos recogidos sean, posteriormente probables de ser analizados. Así, Almeida y Pinto ${ }^{17}$ definen técnicas de investigación como "(...) conjuntos de procedimientos bien definidos y transmisibles, destinados a producir certos resultados en la recogida y tratamiento de la información requerida por la actividad de investigación" (p.78) ${ }^{18}$.

En este estudio, la técnica de recogida de datos utilizada fue la asociación libre de palabras. Esta técnica fue originalmente concebida por Carl Gustav Jung en la práctica clínica, con el objetivo de realizar el diagnóstico psicológico sobre la estructura de la personalidad de los individuos. Se trata de una técnica proyectiva orientada por la hipótesis de que la estructura psicológica del sujeto se torna palpable a través de las conductas, de las reacciones, de las evocaciones, constituyéndose así, en índices reveladores de la personalidad. En cuanto técnica proyectiva, debería actuar sobre la estructura psicológica del sujeto, evidenciándose 
a partir de las cuatro principales condiciones de un test proyectivo: estimular, hacer observable, registrar y obtener la comunicación verbal ${ }^{19}$.

La asociación libre de palabras fue adaptada al campo de la psicologia social por Di Giacomo, en 1981 y, desde entonces, viene siendo cada vez más aplicada en las investigaciones sobre representaciones sociales. Sin embargo, los objetivos de la aplicación de esta técnica difieren de los objetivos iniciales propuestos por Jung. Los investigadores en representaciones sociales buscan identificar las dimensiones latentes de las representaciones a través de la configuración de los elementos que constituyen la red asociativa de los contenidos evocados, en relación a cada estímulo inductor, y por tratarse de una técnica proyectiva, se hacen evidentes los contenidos latentes. Se trata de un instrumento que se apoya sobre un repertorio conceptual en lo que dice respecto al tipo de investigación que permite evidenciar universos semánticos y que colocan en evidencia los universos comunes de palabras cara a los diferentes estímulos y sujetos o grupos. El instrumento se estructura, entonces, sobre la evocación de estímulos inductores que deben ser previamente definidos en función del objeto a ser investigado o al objeto de la representación, tomando siempre en consideración las características de los sujetos empíricos. El instrumento puede tener uno o varios estímulos (verbales o icónicos) escogidos de acuerdo con los criterios de destaque y de coherencia con los objetos de la investigación ${ }^{16}$.

El cuestionario está compuesto por dos partes: una trata sobre las cuestiones en forma de estímulo, y los estímulos presentados a los participantes se relacionan a «DOLOR me hace pensar en...» y «EL PINCHAZO DE LA AGUJA me hace sentir...» y la segunda parte incluye variables de caracterización sociodemográfica (sexo, edad, lugar ocupado en la fratria, número de ingresos anteriores y grado de dolor asociado a la punción venosa) y de evaluación del dolor (escala numérica de evaluación del dolor, constituída por una línea recta, en cuyos extremos está señalado "sin dolor" y "dolor máximo". La línea está dividida en unidades de 0 a 10 y el niño escoge el número que acredita corresponder a la intensidad de dolor que siente. Estas son principalmente utilizadas en niños en edad escolar y en adolescentes) ${ }^{2}$.

\section{Técnica de análisis de datos}

Para identificar los universos semánticos asociados al dolor se efectuó un análisis de correspondencias léxicas (análisis factorial de correspondencias simples - AFC - S) para cada uno de los estímulos. Para efectuar este tratamiento, se recurrió al programa de análisis textual SPAD-T (Sistema Portátil de Análisis de Datos Textuales). El análisis factorial de correspondencias es un proceso a través del cual se determina el menor número de factores que explican una determinada tabla o matriz de correlaciones ${ }^{19}$.

Los métodos de análisis factorial de correspondencias, en particular, son técnicas de estadística descriptiva multivariada que permiten obtener la representación simultánea de dos o más conjuntos de datos que corresponden a variables nominales definidas por las líneas y por las columnas en una determinada matriz de datos, de forma a evidenciar las afinidades entre esas líneas y columnas. El análisis factorial de correspondencias posibilita la organización de los datos en frecuencias, así como la representación gráfica de diferentes conjuntos de inter-relaciones existentes dentro de cada uno de los conjuntos y variables, correspondientes a las líneas y las columnas, y entre ambas, en una tabla de datos ${ }^{15}$.

El programa SPAD-T se aplica a datos obtenidos a través de la asociación libre de palabras, con el objetivo de encontrar la estructura y el contenido de los campos representacionales 
asociados a las palabras estímulo. A través del análisis de correspondencias léxicas, el programa permite obtener una síntesis de la información contenida en la matriz preliminar de datos, a través de la extracción de un determinado número de factores, relevantes para el análisis ${ }^{17}$.

\section{Selección y Caracterización de los Participantes}

En el transcurso de una investigación, es necesario que se tenga presente que será imposible incluir todas las personas de eventual interés para el estudio, consecuentemente, habrán de seleccionarse solo algunas, las cuales constituyen la muestra ${ }^{20}$. El muestreo utilizado en este estudio, es un muestreo por conveniencia. Este es un sub-tipo del muestreo en aleatorio o en probabilística y se define por la selección, de entre toda la población, de los elementos más accesibles ${ }^{21}$. En el caso de este estudio, al pretender recoger los significados asociados al dolor y a la punción venosa, realizando unas prácticas en un servicio de Pediatria de un hospital central, aplicamos la técnica de asociación libre de palabras a los niños que allí estuvieron, a lo largo de dos meses.

La muestra estuvo, entonces, compuesta por cuarenta y tres niños ( $N=43)$ con edades comprendidas entre los 6 y los 12 años, de ambos sexos, que no presentasen déficit cognitivo, sometidos a punción venosa. De los 43 niños inquiridos, $44.2 \%$ eran del sexo masculino y $55.8 \%$ del sexo femenino. La media de edad de los niños es de 10 años (X=9.63), siendo que la mayoría de ellos tenía 12 años $(32.6 \%)$ y los restantes tenían edades comprendidas entre los 6 y los 11 años (6 años - 9.3\%; 7 añnos - 14\%; 8 años 11.6\%; 9 años - 11.6\%; 10 años - 9.3\% e 11 años - 11.6\%). En lo relativo al número de ingresos anteriores, $44.2 \%$ inquiridos refirieron haber estado internados nunca, 30.2\% tuvieron un ingreso anterior a los actuales, 7\% estuvieron internados, anteriormente, dos veces, $14 \%$ tres veces, y los restantes $2.3 \%$ estuvieron ingresados entre cuatro y siete veces respectivamente.

\section{RESULTADOS}

La presentación y discusión de los resultados se hizo basándose en los estímulos: «DOLOR me hace pensar en...» y «EL PINCHAZO DE LA AGUJA me hace sentir...», así como los resultados derivados de la evaluación del dolor asociada a la punción venosa, en niños de edad escolar. De los resultados obtenidos a través de la técnica de asociación libre de palabras, en un primer análisis, fueron retenidas alas palabras con una frecuencia superior a 3 ocurrencias, en cada uno de los estímulos. En una segunda fase del tratamiento de los datos es presentado el análisis factorial de correspondencias simples que utiliza las palabras (variables cualitativas) como unidades de análisis, por lo que es posible atribuir significado a un conjunto de palabras que surgen agrupadas en factores. Las palabras surgen agrupadas en polos positivos y negativos, correspondientes a su proyección en los ejes factoriales, no teniendo, sin embargo, cualquier connotación valorativa en términos de análisis de datos. Los valores numéricos de las contribuciones absolutas que surgen asociados a las palabras corresponden al "peso" que esa palabra tiene en la explicación del factor ${ }^{22}$. En relación a la evaluación del dolor asociado a la punción venosa, se presentan los resultados obtenidos acerca de esta variable, cruzados con las otras variables independientes como el grupo de edad y el número de ingresos.

\section{Representaciones del dolor en el niño}

Para identificar las representaciones asociadas a la palabra dolor en niños en edad escolarsometidos a punción venosa, y a las preguntas de investigación: ¿Qué ideas surgen 
asociadas al estímulo dolor en los niños? ¿Cuáles surgen más frecuentemente? ¿De qué carácter son esas ideas? se hizo el análisis de los resultados obtenidos a partir del estímulo: «DOLOR me hace pensar en...». Fueron, entonces, producidas 98 palabras, 24 (24.5\%) de las cuales son distintas, unas de las otras. Del total de palabras, fueron retidas 87, correspondientes a $88,8 \%$ de las palabras iniciales, de las que 13 son diferentes, conforme se observa en el Cuadro 1.

\begin{tabular}{|c|c|}
\hline Palabra & Frecuencia \\
\hline Sufrimiento & 18 \\
Hospital & 9 \\
Miedo & 9 \\
Llorar & 9 \\
Doler & 6 \\
Estar enfermo & 6 \\
Herida & 6 \\
Ir al médico & 5 \\
No ir a la escuela & 5 \\
Accidente & 5 \\
Voy a mejorar & 3 \\
Nada & 3 \\
Muerte & 3 \\
\hline
\end{tabular}

Cuadro 1 - «DOLOR me hace pensar en...» - Palabras retiradas por orden de frecuencia

En un primer análisis de este cuadro, se puede observar que las palabras más frecuentemente asociadas al dolor (sufrimiento, hospital, miedo y llorar) sugieren que estas son desencadenadoras de sufrimiento, que puede ser expresado a través del llanto y que está, muchas veces, asociado al miedo a la hospitalización. En otro sentido, se ve también que el dolor se asocia a la propia enfermedad o a la existencia de una lesión que pueda ser consecuencia de un accidente. Surge, entonces, la necesidad de "ir al médico". Como es característico en los niños de edad escolar el dolor está también asociado a los beneficios que pueden resultar, como por ejemplo, no tener que ir a la escuela ${ }^{2,3,4}$. Sin embargo, el niño al asociar el dolor a no ir a la escuela también puede atribuirle una connotación negativa ya que en esta fase el niño otorga gran importancia a la escuela y a su papel en el desarrollo de competencias, valores y aprendizajes ${ }^{23}$. Con la creciente comprensión de la importancia de ser y estar saludable, de las consecuencias de enfermar y del significado de la muerte, el niño, en edad escolar, ya une el dolor a la muerte y la voluntad de mejorar rápidamente, sin que haya secuelas, como se puede ver por las expresiones "muerte" y "voy a quedar mejor". Hay incluso niños que asocian el dolor al propio dolor por lo que ya se encuentra subyacente la desvalorización de una posible transgresión, que muchas veces parece ser la causa explicativa del dolor, en niños más pequeños ${ }^{2}$.

A partir del Análisis Factorial de Correspondencias simples de las palabras retenidas, se construyeron tres factores, siendo que los principales elementos de interpretación de estos factores están resumidos en el Cuadro 2 y representados gráficamente en el Gráfico 3.

El primer factor está representado por la palabra NADA (96.6), lo que parece representar algo característico de esta fase de desarrollo de los niños. El hecho de responder que del dolor no se acuerda nada, no quiere decir que no tenga algún tipo de representación del dolor pero no sabe cómo desarrollar la idea o que no tiene certezas suficientes para hacerlo, por lo que prefieren no transmitirla. Según Erik Erikson, el niño en edad escolar, se encuentra en la cuarta edad caracterizada por la dualidad maestría versus inferioridad. En este estadio, es descrito que si el niño no confía en sus capacidades, preferirá no pronunciarse acerca de un asunto sobre el que no tiene certezas, coaccionado por el miedo a equivocarse ${ }^{24}$. 


\begin{tabular}{||l|l|l||}
\hline \multicolumn{2}{|c|}{$\mathbf{N = 4 3}$} \\
\hline Factores & $\begin{array}{l}\text { Coordenadas + } \\
\text { Contribuciones absolutas }\end{array}$ & $\begin{array}{l}\text { Coordenadas - } \\
\text { Contribuciones absolutas }\end{array}$ \\
\hline Nada & Nada (96.6) & \\
\hline F2 \\
Lo Psicológico y lo físico & $\begin{array}{l}\text { Miedo (40.8) } \\
\text { Sufrimiento (5.8) } \\
\text { Doler (5.1) } \\
\text { Muerte (1.1) }\end{array}$ & $\begin{array}{l}\text { Ir al médico (13.3) } \\
\text { Hospital (10.1) } \\
\text { Herida (8.3) } \\
\text { Estar enfermo (5.8) } \\
\text { No ir a la escuela (3.9) }\end{array}$ \\
\hline $\begin{array}{l}\text { F3 } \\
\text { Preocupación con la incapacidad que } \\
\text { deriva del dolor }\end{array}$ & $\begin{array}{l}\text { Voy a mejorar (76.1) } \\
\text { Accidente (3.7) }\end{array}$ & \\
\hline
\end{tabular}

Cuadro 2 - AFC simples - Estímulo 1 “Dolor me hace pensar en...”

$$
\% \mathrm{~F} 1=17.36 \quad \% \mathrm{~F} 2=13.95 \quad \% \mathrm{~F} 3=12.07
$$

En el segundo factor están representadas las ideas que parecen traducir la experiencia dolorosa, revelándose, sin embargo, una oposición entre pensamientos concretos 0 procesos de objetivación del dolor, en las coordenadas negativas, manifestados a través de ideas como "ir al médico"(13.3), "hospital"(10.1), "herida"(8.3), "estar enfermo"(5.8) y "no ir a la escuela"(3.9) y pensamientos difusos o sentimientos expresados ante ese dolor, en las coordenadas positivas, a través de expresiones como "miedo"(40.8), sufrimiento"(5.8), "doler"(5.1) y "muerte" (1.1).

El tercer factor expresa la preocupación del niño por las incapacidades que puedan sobrevenir de una situación de enfermedad y, consecuentemente, de dolor. Se puede constatar esa preocupación a través de expresiones como "voy a mejorar" (76.1) y "accidente" (3.7). Esta situación es bastante común en esta edad. Luísa Barros ${ }^{2}$ refiere, incluso, que el niño en edad escolar demostrará, ciertamente, menos preocupación por el dolor en sí, que por la incapacidad que pueda derivarse de él, o del propio procedimiento que se va a realizar, dado que su desarrollo cognitivo ya le permite tener noción de la importancia de ser y estar saludable, de las consecuencias de caer enfermo y del significado de la muerte.

Del análisis del Gráfico 3 se percibe que en las coordenadas positivas, donde aparecen expresiones como "miedo", "sufrimiento", "daño" y "muerte", están expresados sentimientos y pensamientos de carácter interno caracterizadores del dolor y en las coordenadas negativas, donde encontramos expresiones como "ir al médico", "hospital" y "herida", están presentes algunas dimensiones donde se objetiva el dolor. 


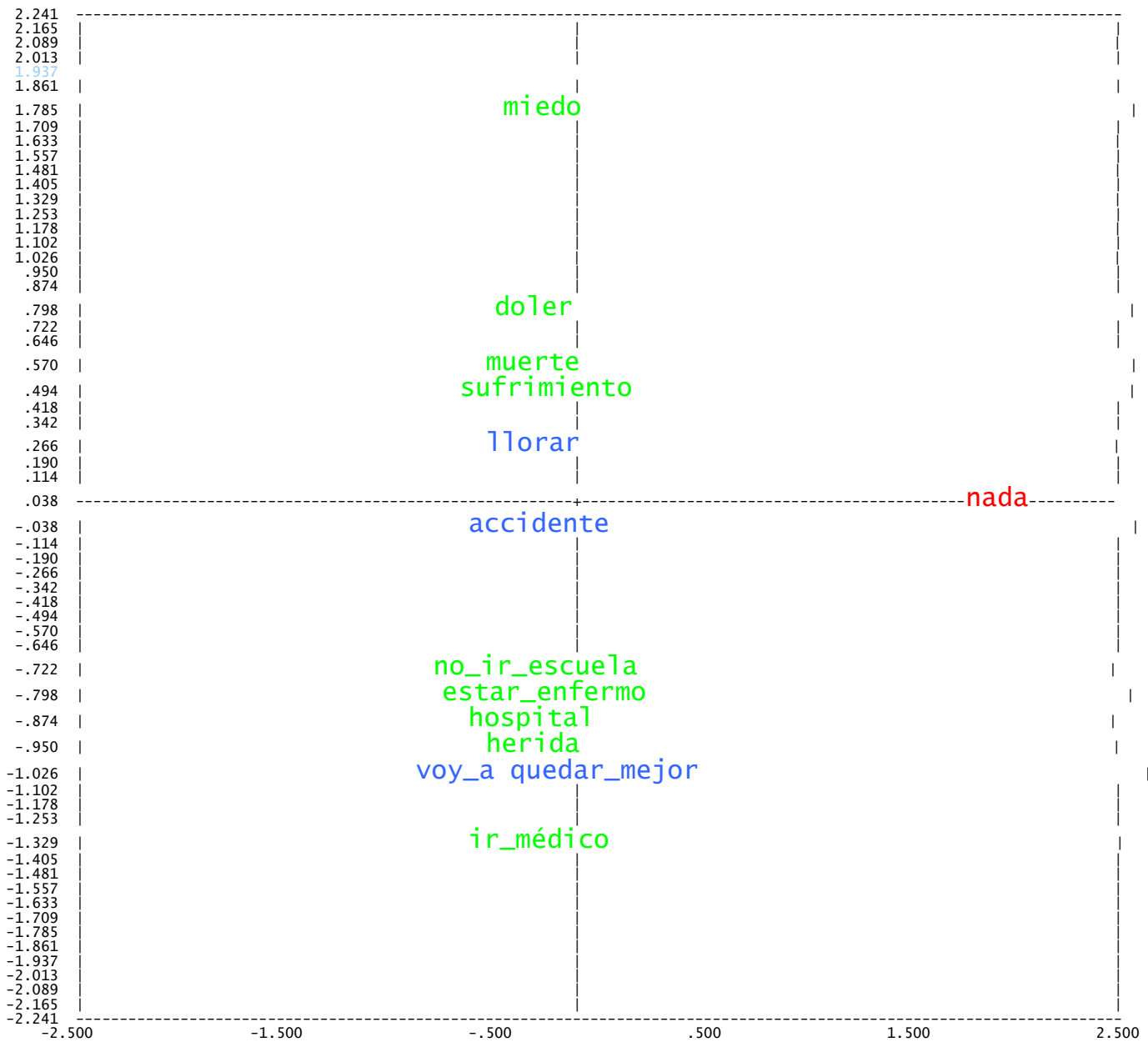

Gráfico 3 - AFC simples de las palabras asociadas a "Dolor hazme pensar en..."

\section{Representaciones de punción venosa en el niño}

En este punto se pretende dar respuesta al objetivo: identificar las representaciones asociadas a la experiencia de punción venosa, en niños de edad escolar, y las siguientes cuestiones de investigación: ¿Qué ideas surgen asociadas a la experiencia de punción venosa en los niños? ¿Cuáles son las más frecuentes? ¿Cuál es la naturaleza de estas ideas?

De esta forma, se hizo el análisis de los datos obtenidos a partir del estímulo «EL PINCHAZO DE LA AGUJA me hace sentir...». Fueron, entonces, producidas 80 palabras, de las que $15(18.8 \%)$ son distintas. De las palabras totales fueron retenidas 73 , correspondientes a $91,25 \%$ de las palabras iniciales. De estas, 9 son diferentes, como se puede observar en el Cuadro 3. 


\begin{tabular}{|c|c|}
\hline Palabra & Frecuencia \\
\hline Dolor & 18 \\
\hline Miedo & 17 \\
\hline Opresión en el corazón & 13 \\
\hline Vergüenza de llorar & 7 \\
\hline Dolor necesario & 5 \\
\hline Picazón & 4 \\
\hline Nada & 3 \\
\hline Valor & 3 \\
\hline Sufrimiento & 3 \\
\hline
\end{tabular}

Cuadro 3 - «EL PINCHAZO DE LA AGUJA me hace sentir...»

- Palabras retenidas por orden de frecuencia

A partir del análisis de este cuadro se puede decir que las palabras más frecuentemente asociadas a la experiencia de punción venosa (dolor, miedo y opresión en el corazón) sugieren que este procedimiento es doloroso y provoca miedo y ansiedad (opresión en el corazón). Sin embargo, muchos niños evitaron manifestar ese dolor por vergüenza o por considerar que es un dolor necesario. Existen, todavía, niños que consideran que es necesario ser valiente, idea que está muy difundida en los niños de esta edad, principalmente de sexo masculino, ya que a partir de los 4/5 años comienzan a evidenciarse expectativas culturales como, por ejemplo, el papel estereotipado de que "los hombres no lloran" ${ }^{2,3,4}$.

Puede constatarse, a través del análisis del cuadro, la subjetividad de la experiencia dolorosa, toda vez que para un mismo procedimiento, existen niños que lo asocian a una picazón y otros que lo asocian a sufrimiento. Como con el estímulo anterior, también fueron construidos tres factores a partir del Análisis Factorial de Correspondencias de las palabras retenidas, en que los principales elementos de interpretación de esos factores están presentados en el Cuadro y Gráfico 4.

El primer factor, a semejanza de lo que sucedió para el estímulo «DOLOR me hace pensar en...», es también representado por la palabra NADA (95.9), lo que puede ser justificado por el miedo a él y de la inferioridad que de ahí pueda venir, como explicamos anteriormente.

El segundo factor representa la aceptación de los niños en relación al dolor asociado a la punción venosa, pues la palabra conmayor peso explicativo para ese factor refiere que este es un "dolor necesario"(71.4), a pesar del "miedo"(5.9) y de la ansiedad ("opesión en el corazón" - 5.9) inherentes al procedimiento.

El tercer factor expresa los mecanismos de enfrentamiento del niño en relación a la punción venosa, contraponiendo, por un lado el "dolor"(4.6) y la "vergüenza de llorar"(21.2) y por otro el "valor" (66.1). 


\begin{tabular}{||l|l|l|}
\hline N=43 & $\begin{array}{l}\text { Coordenadas }+ \\
\text { Factores }\end{array}$ & $\begin{array}{l}\text { Coordenadas - } \\
\text { Contribuciones absolutas }\end{array}$ \\
\hline $\begin{array}{l}\text { F1 } \\
\text { Nada }\end{array}$ & Nada (95.9) \\
\hline $\begin{array}{l}\text { F2 } \\
\text { Aceptación }\end{array}$ & $\begin{array}{l}\text { Opresión en el corazón } \\
(5.9) \\
\text { Miedo (5.9) }\end{array}$ & $\begin{array}{l}\text { Dolor necesario (71.4) } \\
\text { Picazón (4.3) }\end{array}$ \\
\hline $\begin{array}{l}\text { F3 } \\
\text { Enfrentamiento }\end{array}$ & $\begin{array}{l}\text { Vergüenza de llorar } \\
(21.2) \\
\text { Dolor (4.6) }\end{array}$ & Valentía (66.1) \\
\hline
\end{tabular}

Cuadro 4 - AFC simples - Estímulo 2 "El pinchazo de la aguja me hace sentir..."

$\% \mathrm{~F} 1=21.63 \quad \% \mathrm{~F} 2=17.29 \quad \% \mathrm{~F} 3=14.60$

En el análisis del Gráfico cuatro, atendiendo a la posición de las palabras, en lo que dice respecto a las coordenadas positivas y negativas, se percibe que como en las coordenadas negativas aparecen expresiones relacionadas con el dolor del pinchazo en sí, como "dolor", "dolor necesario", "picazón", en las coordenadas negativas están expresadas ideas de foro más íntimo e individual como es el caso del "miedo", de la vergüenza de llorar y de la opresión en el corazón. Estas expresiones son, también, reveladoras de los sentimientos provocados en el niño, al pensar que será sometido a un procedimiento invasivo, como es el caso de la punción venosa. 


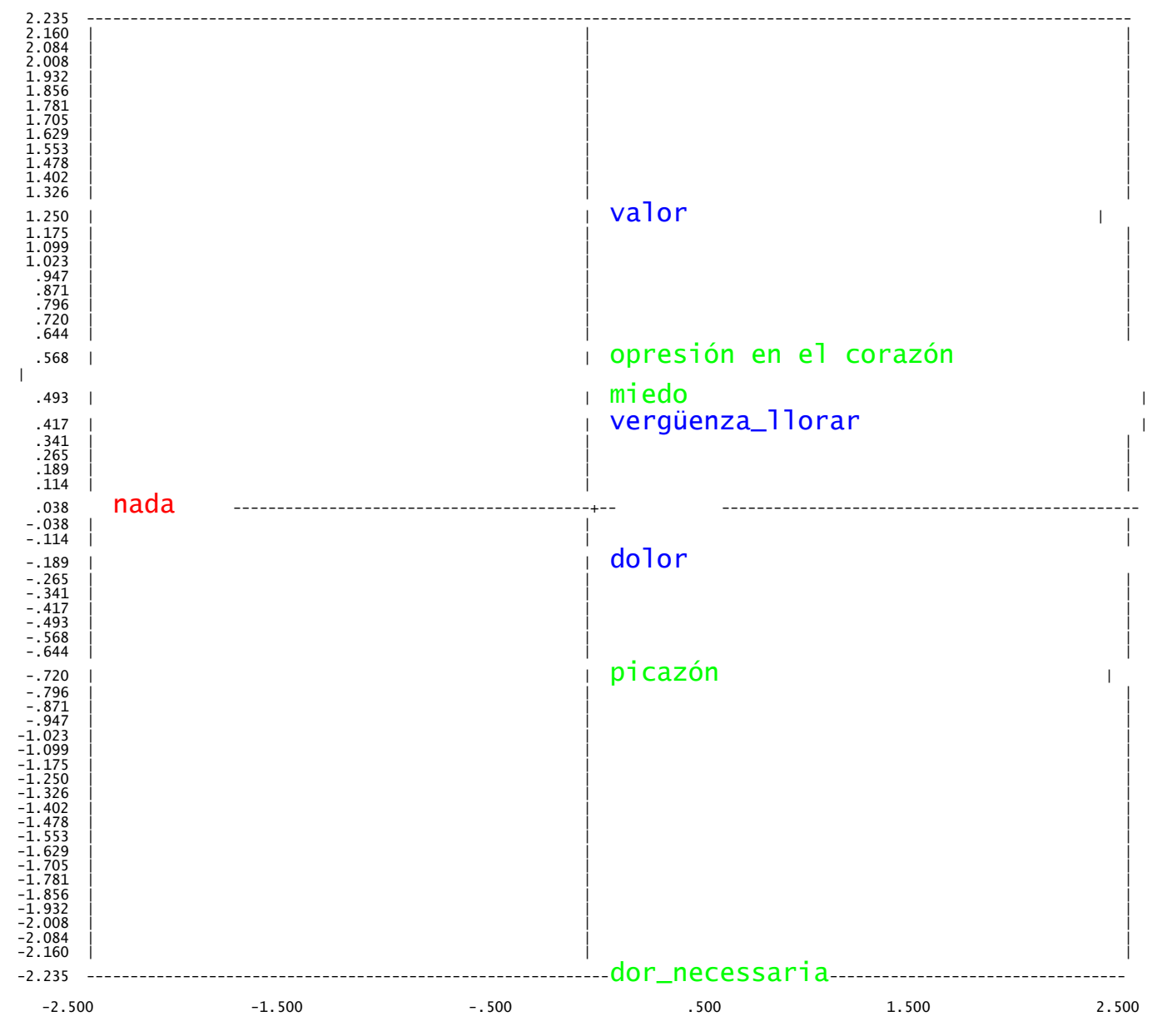

\section{Gráfico 4 - AFC simples de las palabras asociadas a "El pinchazo de la aguja me hace sentir..."}

\section{Dolor asociado a la punción venosa en el niño}

Al analizar los datos relativos a la evaluación del dolor asociado a la punción venosa verificamos que el grado de dolor asociado a la punción venosa en los 43 niños de nuestro estudio osciló entre un grade de dolor mínimo de 1 , referido por $2.33 \%$ de los niños y el grado de dolor máximo referido fue de $9.3 \%$. La media del dolor asociado a punción venosa, evaluada en estos niños se sitúa en los 5.23.

En el gráfico 5 se procedió al cruzamiento de las variables grupo etario, media de grado de dolor asociado a punción venosa y número de ingresos anteriores. La media corresponde al punto señalado con un círculo, siendo que la amplitud de cada representación gráfica muestra la varianza de las respuestas en relación a la media. Como se observa en los niños que tuvieron entre cero y dos ingresos anteriores, a pesar de pertenecer a grupos etarios diferentes, la media y la varianza en relación a la media es muy semeljante. Ya en los niños con tres o más ingresos anteriores las medias son diferentes, así como la varianza es superior lo que remite a la importancia que las experiencias anteriores tienen en el grado de dolor experienciado. Luisa Barros escribe que aunque la edad sea un dato crucial para la forma como el niño experiencia y manifiesta el dolor, aisladamente representa muy poco, necesitando de otros factores que conjuntamente con ella tengan sentido. De esta forma hay que tener en cuenta elementos como experiencias pasadas, ya sea directamente o con las 
personas más próximas al niño. Estas serán determinantes para que el niño construya, en el seno de la cultura familiar, su noción de sufrimiento y defina formas de evitarlo ${ }^{2}$

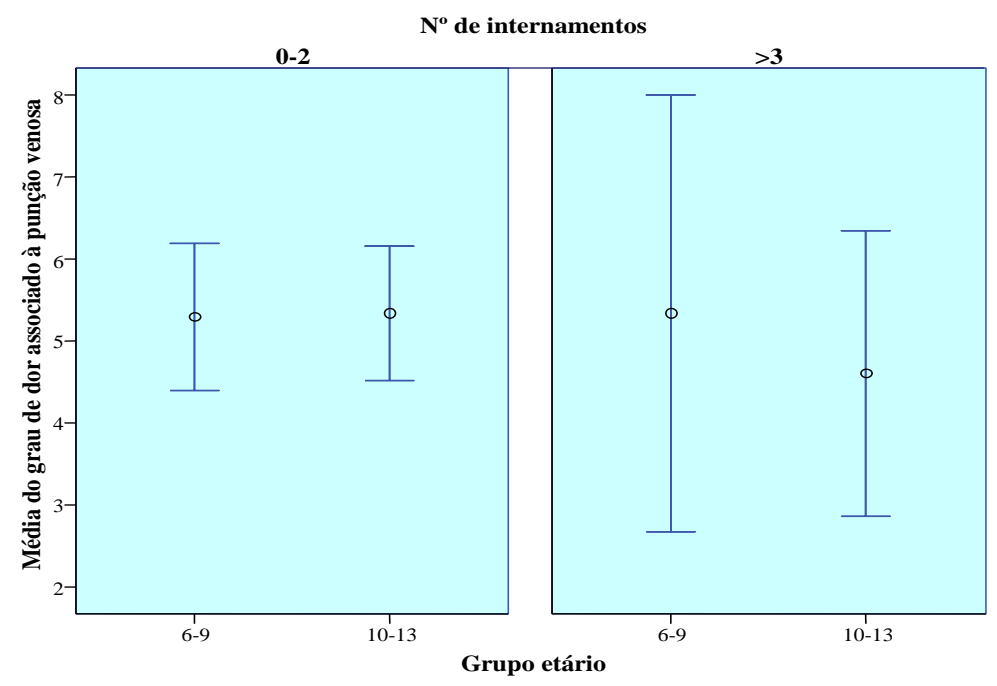

Gráfico 5 - Participantes por grupo etário/ no de ingresos y grado de dolor asociado a la punción venosa (média)

\section{CONCLUSIONES}

En este estudio, se trató de conocer las representaciones del dolor en los niños, en edad escolar, que hubiesen sido sometidos a punción venosa y en este sentido fue elaborado un instrumento de recogida de datos, recurriéndose a la asociación libre de palabras con dos estímulos: «DOLOR me hace pensar en...» y «EL PINCHAZO DE LA AGUJA me hace sentir...». Se consideró también que la evaluación del dolor asociado al procedimiento de punción venosa sería importante para objetivar un concepto tan relativo e individual como el dolor.

En este sentido, en relación a las representaciones que los niños hacen del dolor se concluye que este es considerado, frecuentemente, un factor desencadenante de sufrimiento, exteriorizado muchas veces a través del llanto y asociado al miedo a la hospitalización. Se concluye también que los niños asocian dolor a enfermedad, a estar enfermo y a ir al médico, como forma de procurar alivio para el dolor y para la enfermedad. En otra perspectiva se verifica que el dolor también puede asociarse tanto a la preocupación de no poder realizar las mismas actividades que los otros niños, por ejemplo, no poder ir a la escuela, como a una "disculpa" para no tener que realizar una actividad menos agradable. Los niños asocian también el dolor a la muerte y a la incapacidad, demostrando una comprensión creciente de la importancia de ser y estar sano, de las consecuencias de caer enfermo y del significado de la muerte. También en realción a las representaciones que el niño hace del dolor se puede concluir que estas revelan una oposición entre pensamientos concretos, ligados a las manifestaciones físicas del dolor y pensamientos difusos o sentimientos expresados ante ese dolor, como el miedo, el sufrimiento y la muerte.

Las ideas más frecuentemente asociadas a la punción venosa, permiten concluir que este procedimiento es doloroso y provoca miedo y ansiedad en los niños. Sin embargo, muchos evitaron tener comportamientos sugestivos de dolor por vergüenza de ser considerados más débiles o por entender que es un dolor necesario. De ahí el hecho de que algunos niños 
asocien el dolor al valor que es necesario tener para soportarlo. A partir de los resultados obtenidos puede, también, concluirse que el dolor es una realidad altamente subjetiva, tal como se ha referido a lo largo de todo el trabajo, ya que las concepciones de dolor del niño varian ya en intensidad, ya en términos dimensionales. Algunos niños asocian el dolor del pinchazo a picazón, otros lo asocian al propio dolor, así como al sufrimiento. Por otro lado, también se puede constatar la individualidad del dolor a través de las dimensiones valorizadas. Mientras algunos niños se centran en factores de orden físico, otros valoran factores de orden abstracto como el miedo y la ansiedad.

En relación al dolor asociado a punción venosa interesa realzar los factores que pueden condicionar diferentes grados de dolor relacionado con un mismo procedimiento. A través del análisis de los datos obtenidos se concluye que las experiencias anteriores tienen un gran peso en la variación del grado de dolor. Esto puede ser explicado por el hecho de haber niños que nunca tuvieron contacto con el medio hospitalario y con los procedimientos que allí son ejecutados, como es por ejemplo la punción venosa. En estos casos el miedo y la ansiedad están aumentados por lo desconocido. Es por ello determinante aclarar al niño sobre lo que va a pasar, transmitiéndole de esta forma seguridad. Otro factor determinante para la atribución de significado al procedimiento doloroso y consecuentemente el dolor que acarrea está relacionado con la edad. Los niños mayores evaluaron el dolor inherente a la punción venosa en grados más bajos que los niños más pequeños, por lo que se puede concluir que su madurez intelectual ya les permite comprender que la punción venosa no causará daños irreversibles y que es una situación pasajera.

\section{REFERÊNCIAS BIBLIOGRÁFICAS}

1. Caetano, Ana Cristina Tadeia Ferreira; et al (2003), "Reflexões sobre a actuação do

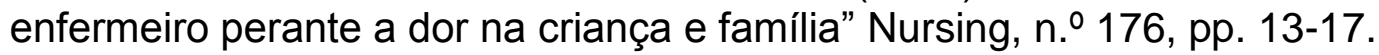

2. Barros, Luísa (2003), Psicologia Pediátrica - Perspectiva Desenvolvimentalista, Lisboa, Climepsi Editores.

3. Sorensen e Luckmann (1998), Enfermagem Fundamental - Abordagem psicofisiológica, Lisboa, Lusodidacta.

4. Whaley, Lucille F. e Wong, Donna L. (1999), Enfermagem Pediátrica, Rio de Janeiro, Editora Guanabara Koogan.

5. Gaffney, Anne (1993), "Cognitive Development Aspects of Pain in School - Age Children" in Schechter, Berde e Yaster (eds), Pain in Infants, Children and Adolescents, Baltimore: Williams\&Williams, 75-85.

6. Casey, Anne (1993), "Development and use of Partnership Model of Nursing Care" in Glasper e Tucher, Advances in child health nursing, Londres, Scutari, pp. 193 - 238.

7. Bowden, Vicky R. e Greenberg, Cindy Smith (2005), Procedimentos de enfermagem pediátrica, Rio de Janeiro, Guanabara Koogan.

8. Mansson, Marie Edwinson e Dykes, Anna-Karin (2004), "Practices for Preparing Children for Clinical Examinations and Procedures in Swedish Pediatric Wards", Pediatric Nursing, Vol.30, no 3, pp. 182-187.

9. Jodelet, Denise (2001), "Representações Sociais: Um domínio em expansão", in Denise Jodelet (org), As Representações Sociais, Rio de Janeiro, Editora da Universidade do Estado do Rio de Janeiro, pp. 17-44.

10.Vala, Jorge (2004), "Representações sociais e psicologia social do conhecimento quotidiano", in Jorge Vala e Maria Benedicta Monteiro (coord.), Psicologia Social, Lisboa, Fundação Calouste Gulbenkian, pp 457-502. 
11.Sá, Celso Pereira de (1998), A Construção do Objecto de Pesquisa em Representações Sociais, Rio de Janeiro, Editora da Universidade do Estado do Rio de Janeiro.

12. Moscovici, Serge (2003), Representações Sociais - Investigação em psicologia social, Petrópolis, Editora Vozes.

13. Abric, Jean-Claude (2000), "A Abordagem Estrutural das Representações Sociais", in Antónia Silva Paredes Moreira e Denize Cristina de Oliveira, Estudos Interdisciplinares de Representação Social, Goiânia, AB Editora, pp. 27-38.

14.Wagner, Wolfgang (2000), "Sócio-Génese e Características das Representações Sociais", in Antónia Silva Paredes Moreira e Denize Cristina de Oliveira, Estudos Interdisciplinares de Representação Social, Goiânia, AB Editora, pp. 3-25.

15. Oliveira, Abílio (1995), Percepção da Morte: Realidade Interdita, Tese de Mestrado em Psicologia Social Especialidade Cognição Social, Instituto Superior de Ciências de Trabalho e da Empresa, Lisboa.

16. De Rosa, Annamaria Silvana (2005), "A rede associativa: Uma técnica para captar a estrutura, os conteúdos e os índices de polaridade, neutralidade e estereotipia dos campos semânticos relacionados com as representações sociais", in Antonia Silva Paredes Moreira; Brígido Vizeu Camargo Jorge Correia Jesuíno, Sheva Maia Nóbrega (org.), Perspectivas Teorico-Metodológicas em Representações Sociais, Paraíba, Editora Universitária - UFPB, pp. 61-127.

17. Almeida, João Pereira de e Pinto, João Madureira (1990), Investigação nas ciências sociais, Lisboa, Editorial Presença.

18. Nóbrega, Sheva Maia da e Coutinho, Maria da Penha de Lima (2003), "O Teste de Associação Livre de Palavras", in Maria da Penha de Lima Coutinho; Aloísio da Silva Lima; Francisca Bezerra de Oliveira e Maria Lucinete Fortunato (orgs), Representações Sociais: Abordagem Interdisciplinar, Paraíba, João Pessoa Editora Universitária, pp. 67-77.

19.Pereira, Francisco José Costa (2005), "Análise de Dados Qualitativos Aplicados às Representações Sociais", in Antonia Silva Paredes Moreira; Brígido Vizeu Camargo Jorge Correia Jesuíno, Sheva Maia Nóbrega (orgs), Perspectivas TeoricoMetodológicas em Representações Sociais, Paraíba, Editora Univeritária - UFPB, pp. 25-60.

20. Hicks, Carolyn M (2006), Métodos de Investigação para Terapeutas clínicos Concepção de projectos de aplicação e análise, Loures, Lusociência.

21.Maroco, João (2007), Análise Estatística com Utilização do SPSS, Lisboa, Edições Sílabo.

22. Gaspar, Maria Filomena et al (2000), "Percepção e imagens do papel do Enfermeiro Chefe", Pensar em Enfermagem, vol 4, ㄲo2, 2ㅇsem, pp.4-11.

23. Monteiro, Manuela e Santos, Milice Ribeiro dos (2002), Psicologia, Porto, Porto Editora. 